\title{
Concurrent Multiscale Modeling of Embedded Nanomechanics
}

\author{
R. E. Rudd
}

This article was submitted to

Materials Research Society Spring Meeting 2001, San Francisco, California, April 16-20, 2001

\section{April 13, 2001}

U.S. Department of Energy

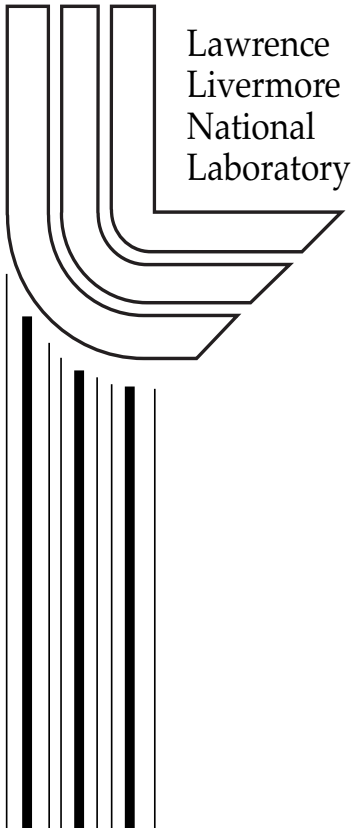




\section{DISCLAIMER}

This document was prepared as an account of work sponsored by an agency of the United States Government. Neither the United States Government nor the University of California nor any of their employees, makes any warranty, express or implied, or assumes any legal liability or responsibility for the accuracy, completeness, or usefulness of any information, apparatus, product, or process disclosed, or represents that its use would not infringe privately owned rights. Reference herein to any specific commercial product, process, or service by trade name, trademark, manufacturer, or otherwise, does not necessarily constitute or imply its endorsement, recommendation, or favoring by the United States Government or the University of California. The views and opinions of authors expressed herein do not necessarily state or reflect those of the United States Government or the University of California, and shall not be used for advertising or product endorsement purposes.

This is a preprint of a paper intended for publication in a journal or proceedings. Since changes may be made before publication, this preprint is made available with the understanding that it will not be cited or reproduced without the permission of the author.

This report has been reproduced directly from the best available copy.

Available electronically at http://www.doc.gov/bridge

Available for a processing fee to U.S. Department of Energy

And its contractors in paper from

U.S. Department of Energy

Office of Scientific and Technical Information

P.O. Box 62

Oak Ridge, TN 37831-0062

Telephone: (865) 576-8401

Facsimile: (865) 576-5728

E-mail: reports@adonis.osti.gov

Available for the sale to the public from

U.S. Department of Commerce

National Technical Information Service

5285 Port Royal Road

Springfield, VA 22161

Telephone: (800) 553-6847

Facsimile: (703) 605-6900

E-mail: orders@ntis.fedworld.gov

Online ordering: http:/ / www.ntis.gov/ordering.htm

\section{OR}

Lawrence Livermore National Laboratory

Technical Information Department's Digital Library

http: / / www.llnl.gov/tid/Library.html 


\section{Concurrent Multiscale Modeling of Embedded Nanomechanics}

Robert E. Rudd

Lawrence Livermore National Laboratory

Condensed Matter Physics, L-415

Livermore, CA 94551 USA

\section{ABSTRACT}

We discuss concurrent multiscale simulations of dynamic and temperature-dependent processes found in nanomechanical systems coupled to larger scale surroundings. We focus on the behavior of sub-micron Micro-Electro-Mechanical Systems (MEMS), especially micro-resonators. The coupling of length scales methodology we have developed for MEMS employs an atomistic description of small but key regions of the system, consisting of millions of atoms, coupled concurrently to a finite element model of the periphery. The result is a model that accurately describes the behavior of the mechanical components of MEMS down to the atomic scale. This paper reviews some of the general issues involved in concurrent multiscale simulation, extends the methodology to metallic systems and describes how it has been used to identify atomistic effects in sub-micron resonators.

\section{INTRODUCTION}

The mechanics and mechanical dynamics of nanoscale systems is often very different from their macroscopic counterparts [1]. Large enough systems behave as if they were composed of continuous media. Whether a particular system is large enough depends on how its size compares to the largest microscopic features of the material. Microstructure matters, or at least it can matter if the length scale of the microstructure is comparable to macroscopic length scales, such as those set by strain gradients. In pure single crystals, materials already begin to appear continuous in many ways at the length scale of tens of lattice spacings. Nevertheless, at sufficiently small length scales the fact that the material is composed of atoms and is not a continuous medium will become apparent. This effect is one reason that mechanics at the nanoscale is not simply macroscopic mechanics reduced in size. Nanomechanics is a separate, and as yet only vaguely understood, field.

Another distinction of nanomechanics is the fact that nanoscale systems often have a large surface area-to-volume ratio. In general, this ratio grows as the size of a system is reduced, and the emergence of surface effects in sub-micron systems has become a recurrent theme in nanoscience. For example, surface effects have a large impact on the structure and morphology of quantum dots [2].

In this Article, we examine some of the new phenomena of nanomechanics based on our computations and in light of recent experimental results from other groups. We also describe new computational tools we have developed in order to compute nanomechanical effects. Of particular interest to us are nanomechanical systems that are strongly coupled to their surroundings, which are typically micron scale or larger. The nanoscale system may be on the surface of a substrate to which it is coupled, or it may be completely surrounded by material. This particular kind of multiscale system, whether on the surface 


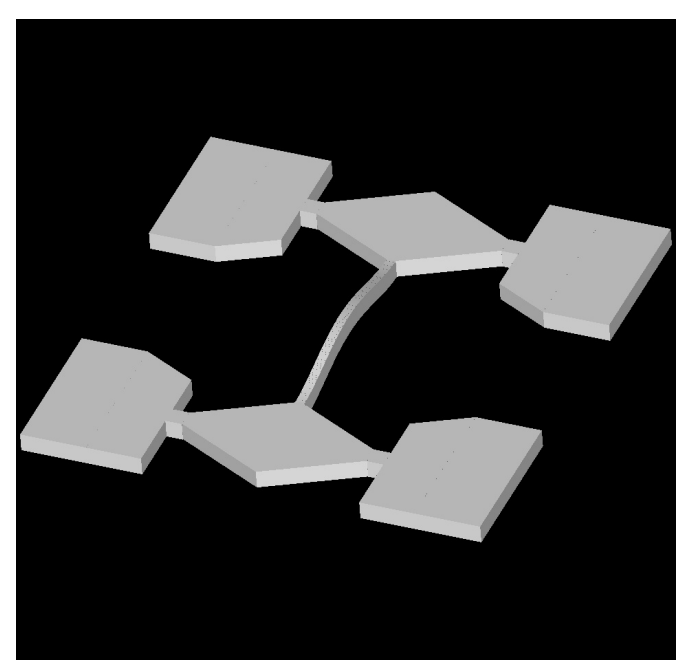

Figure 1: Three dimensional model of the microresonator in its fully deflected state. The geometry is modeled on a device fabricated by Roukes and coworkers [4].

or in the bulk, is what we term embedded nanomechanics. It is a situation that is arising increasingly in systems under study.

The growing interest in the behavior of materials in the sub-micron and nanometer regimes has been sparked by the rapidly expanding set of tools available for fabrication and characterization at these length scales and the enormous potential for new devices and applications that is beginning to be realized. For example, resonators and filters for gigahertz communications applications are now being developed by fabrication of oscillators directly on the semiconductor wafer that contains the control and processing circuitry [3]. Moving parts on computer chips such as this form the basis of the technology of Micro-Electro-Mechanical Systems (MEMS). The extraordinary feature of the gigahertz resonators is that the width of the oscillating semiconductor bar is only tens of nanometers [4-6]; i.e. a device on the order of one hundred atoms across (see Fig. 1). These devices are fabricated by electron beam lithography, one of the emergent fabrication techniques.

Another example is the semiconductor islands that form in a variety of heteroepitaxial systems, and may be used in solid state quantum dot applications [7]. These structures too are on the order of one hundred atoms across, but in this case they form by self-assembly in a deposition process such as chemical vapor deposition (CVD) or physical vapor deposition (PVD). They have been characterized by photoluminescence and atomic force microscopy (AFM), in addition to other forms of scanning probe microscopy [2]. Other examples can be found in fields as diverse as dynamic fracture, where nanoscale voids grow and interact through long range deformations to form the fracture surface [8], and biomechanics, where nanoscale AFM tips induce both nanoscale and micron scale deformations of living cells [9].

Nanoscale and sub-micron systems pose an exciting challenge to modeling for several reasons. Firstly, the existing complement of experimental tools for characterization of these systems can offer only an incomplete picture of the behavior of the material. Often the phenomena of interest are only partially characterized by the available tools, and modeling can fill out this picture. We discuss below how this is being done to understand the 
nanoscale dissipation processes that determine the quality of sub-micron resonators. Secondly, the length scale is small enough that it is often possible to develop models of nanoscale systems from first principles. By limiting the empirical input into the model, the model become more robust in principle and it gains reliability across a broader range of systems. This is especially important due to the limitations of experimental characterization. Finally, while devices such as the gigahertz resonator are based on the geometry of larger devices, systems which are well understood and described using conventional engineering models, new physics may be encountered in the process of miniaturization which modifies or even invalidates the design rules devised at the larger scales.

It is well known that new physics may enter upon miniaturization that sets limits on the way the device can be fabricated, such as diffraction limits in lithography, but there may also be new physics that causes the operational behavior of the device to depart from expectations. Until this physics is understood it forms an obstacle to device development and optimization. The regions of the system in which atomistic effects are important are usually limited to, or typified by, a very small part of the overall system. Outside this region conventional continuum theory works well. This suggests that it would be possible to manage the expense of a first principles technique since it would be used in an extremely small volume. In the systems we consider, what we mean by a first principles technique is an atomistic simulation using an empirical potential, although in other contexts it could be a more fundamental model. Empirical potentials allow the study of millions of atoms, in some cases even thousands of millions, and this encompasses the most important region of many sub-micron devices, such as the oscillating bar of a micro-resonator; however, it alone does not give a complete description of the behavior of the system. The difficulty is that the small scale region interacts with its surroundings. For example, long range strain fields may extend out from the small-scale region into the large-scale region. This dynamic coupling must be described in order to have a robust model.

The methodology we have used is to embed atomistics into a finite element model of continuum mechanics. The two traditionally distinct techniques are coupled together and run concurrently. This captures both the small scale (atomistic) physics and the large scale (continuum) physics. It is an example of concurrent multiscale modeling [10], and this computational approach is well suited to the study of embedded nanomechanics.

In this Article, we describe how the methodology is implemented. A more complete description has been given elsewhere [10], but there have been some recent advances that we describe. In particular, we have extended the technique to work for many-body empirical potentials such as the Embedded Atom Model [11,12] and the Finnis-Sinclair Model [13]. We then describe some results from our studies of MEMS systems, and how they are related to several important new experiments.

\section{THEORY}

Fig. 1 shows the microresonator geometry we have used based on a device fabricated by the Roukes group [4]. In the actual device the bar in the middle and the diamond-shaped tabs at either end have been released from the underlying substrate by etching. Only the four corner tabs are anchored to the substrate. The entire device has 


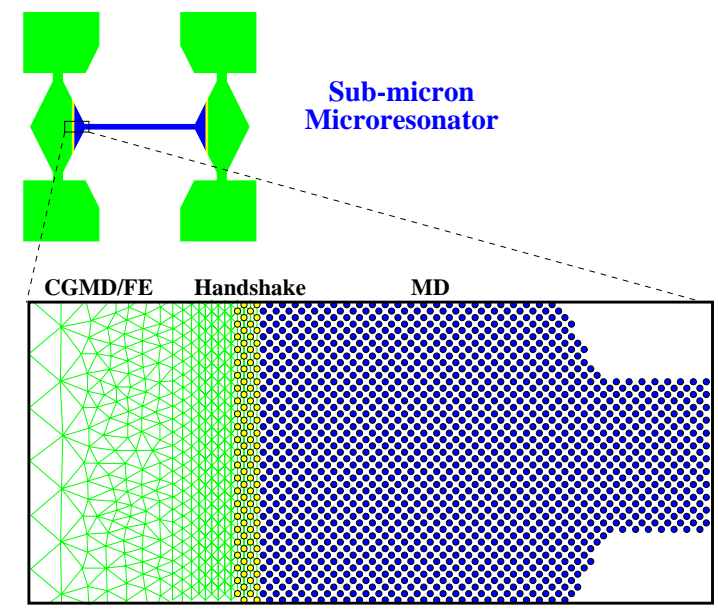

Figure 2: The decomposition of the microresonator system into molecular dynamics (MD) and finite element (FE) or coarse-grained molecular dynamics (CGMD) regions. A narrow handshaking region is used to couple the FE and MD regions; in the case of CGMD, the cross-over to atomistics for atomic-sized cells is completely smooth and no handshaking region is required. The multiscale technique directs the computer power to the important small-scale regions of the system. The basic implementation of this multiscale methodology on parallel supercomputer architectures is described in detail in Ref. [10].

been fabricated from a single crystal.

Two regions are indicated. The oscillating bar in the middle is the region where atomistic processes are to be studied. The peripheral regions are there in our simulation to support the strain fields that extend from the bar. They are continuous in character. The multiscale methodology uses molecular dynamics (MD), a classical atomistic methodology [14], to model the bar and a small region of the tabs at the two ends. The remainder of the system, certainly the vast majority of the volume, is treated with finite element $(\mathrm{FE})$ analysis based on a Galerkin discretization of linear elastic theory [15]. The parameters are the density and elastic constants chosen to match those of the MD region. This is shown in Fig. 2.

In this Section we describe how the coupling between the FE and MD regions is done in detail, and we give some indications of the theoretical motivation to implement the coupling in this fashion. The idea for coupling FE and MD regions in a dynamical simulation was introduced by Gumbsch and coworkers [16], although technical difficulties prevented the development of their technique. Phillips and coworkers [17] also introduced a FE/MD methodology which they have called the Quasicontinuum Technique, but it was developed for lattice statics rather than a dynamical simulation. The methodology we describe here is based on the ideas of Broughton, et al [18], who developed a minimal coupling methodology for dynamical simulation that linked Stillinger-Weber silicon MD [19] to two-dimensional linear elastic FE in order to study crack propagation. In fact, they went further and linked the MD to an atomistic region governed by forces computed using a tight-binding (TB) quantum-mechanical model, but we have no need for a TB region in 
the systems we describe here. Concurrently with Broughton and coworkers, we developed a technique that couples silicon [19] and quartz [20] atomistics to three-dimensional linear elastic FE for MEMS simulation $[21,22,10]$.

Here we reformulate the ideas used previously in the FE/MD coupling in a formalism with a broader applicability. In particular, we present a methodology that is suitable for coupling metallic empirical potentials, such as embedded atom (EAM) [11] or Finnis-Sinclair [13] potentials, to a three dimensional linear elastic finite element model. The formulation of the FE/MD coupling in silicon is not immediately applicable to these systems because of the difference in the form of the many-body interactions; however, the silicon coupling is a special case of the coupling we present here, as is explained below.

In order to construct the concurrent multiscale model, we follow a slight generalization of the finite element paradigm. The first step is to tessellate the system with elements comprising a mesh. The mesh is used to discretize the displacement field in the continuum region, such that a vector displacement variable is located at each node of the mesh. Next, equations of motion for the nodal displacements are introduced. In the FE region these are just the usual finite element equations of motion, the discrete analogs of the constitutive equations that describe how the material behaves in the continuum. In the MD region they are just the usual atomistic equations of motion that describe empirically the binding between neighboring atoms. At the FE/MD interface, new equations of motion must be introduced. The equations of motion also determine the range of the interactions, and once this is set, a domain decomposition is introduced in order to map the system onto the parallel architecture of a supercomputer. The equations of motion are a set of coupled ordinary differential equations, essentially Newton's equations $\vec{F}=m \vec{a}$, and an integrator must be introduced to evolve the system in time. Then once the initial and boundary conditions are enforced, the model is completely specified. Typically, we use periodic boundary conditions on the outer FE boundary, and we take an unstrained initial configuration with random velocities consistent with a Boltzmann distribution. The system is evolved in time for some period with a thermostat turned on in order to bring it to thermal equilibrium. Then typically the thermostat is turned off, and the thermalized state is used as the initial configuration for the subsequent evolution of the system.

We now consider the steps of the construction of the model in more detail starting with the definition of the mesh. Consider an atomistic representation of the entire system of interest. In particular, suppose we know the equilibrium positions of all of the atoms encompassed in the system. We choose a surface that divides the system into MD and FE regions such that the surface is smooth and it does not pass through any of the equilibrium atomic positions. We call this the fiducial surface. In the FE region we introduce a mesh which is in general coarse in the far periphery and refined down to the atomic scale as it approaches the MD region. The mesh extends past the fiducial surface as indicated in Fig. 3. In some cases it is convenient to refine the mesh such that the nodes of the mesh are in one-to-one correspondence with the equilibrium positions of the atoms at the FE/MD interface, but we do not assume that is the case at this point. We do position the nodes at the equilibrium sites, but it is not necessary that every site has a node. We use a mesh generation algorithm that we have developed that respects the underlying atomic lattice, as explained in Ref. [23].

As stated above, the usual FE and MD equations of motion are used in the FE and 


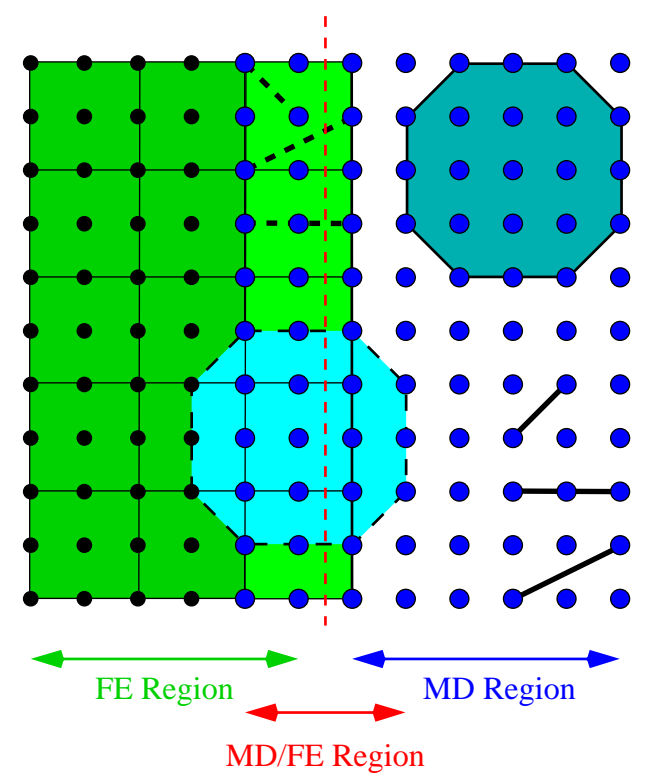

Figure 3: The coupling between finite elements and molecular dynamics in the case of a manybody MD potential such as the Embedded Atom Model. The clusters depict a group of atoms within the range of the potential of a single atom. Each such cluster that crosses the fiducial MD/FE interface enters the handshaking Hamiltonian as a whole, with an overall weight. Similarly, each FE cell crossing the interface enters as a whole, appropriately weighted. See the text for details of how the weights are assigned.

MD regions, respectively. It is only in the interfacial FE/MD region that new equations of motion must be introduced. The definition of the kinetic energy is straight-forward. The lumped mass approximation is used on the FE side of the fiducial surface, so that the mass is effectively concentrated at the nodes of the mesh

$$
\begin{aligned}
E_{\text {kinetic }} & =\frac{1}{2} M_{i j} \dot{\vec{u}}_{i} \cdot \dot{\vec{u}}_{j} \\
& =\frac{1}{2} m_{i} \dot{\vec{u}}_{i} \cdot \dot{\vec{u}}_{i}
\end{aligned}
$$

where $M_{i j}=m_{i} \delta_{i j}$ is the lumped mass matrix, $\vec{u}_{i}$ is the displacement at node $i$, and repeated indices are summed over the three spatial dimensions. According to the lumped mass approximation [15], $m_{i}$ is given by the density of the material times the volume of the Voronoi cell about node $i$. The usual MD kinetic energy is used on the MD side of the fiducial surface

$$
E_{\text {kinetic }}=\frac{1}{2} m_{\alpha}\left|\dot{\vec{x}}_{\alpha}\right|^{2}
$$

where $m_{\alpha}$ and $\vec{x}_{\alpha}$ are the mass and position of atom $\alpha$, respectively. Note that the state of the system is completely determined by the coordinate variables $\left\{\vec{x}_{\alpha}\right\}$ and $\left\{\vec{u}_{i}\right\}$, and their conjugate momenta. 
The lumped mass approximation is appropriate in the atomic limit where each node is associated with an individual nucleus, and this is where the mass is concentrated. The lumped mass approximation also allows explicit time integration, as in molecular dynamics, and this permits greater uniformity throughout the simulation, a point to which we return below. In principle, it is possible, and perhaps even appropriate, to use the full distributed mass matrix in the $\mathrm{FE}$ region, but we have not explored its ramifications.

The definition of the potential energy in the interfacial region is more challenging because of the finite range of the interactions and their many-body character. The case of the pair potentials is the most straight-forward, so we consider it first. This prescription has been described elsewhere [10], and it is based on the ideas Broughton, et al [18], developed for a (100) FE/MD interface in Stillinger-Weber silicon. Basically, we use mean forces at the interface that average the forces on the two sides. There is some ambiguity in how the energy and the corresponding forces are apportioned among the sites, and we have made choices that ensure the proper structural mechanics and sensible dynamics. In particular, we optimize the elastic constants and minimize the elastic wave coefficient of reflection, while keeping the interface as small as possible.

Let the FE contribution to the interface consists of those cells that cross the fiducial surface. We denote the volume occupied by these cells as $\Omega$. Then the potential energy at the interface is given by

$$
\begin{aligned}
E_{\text {potential }}^{\mathrm{FE} / \mathrm{MD}} & =\frac{1}{2} E_{\text {potential }}^{\mathrm{FE}}(\Omega)+\frac{1}{2} E_{\text {potential }}^{\mathrm{MD}}(\Omega) \\
E_{\text {potential }}^{\mathrm{FE}}(\Omega) & =\sum_{i, j \in \Omega} \frac{1}{2} \vec{u}_{i} K_{i j} \vec{u}_{j} \\
E_{\text {potential }}^{\mathrm{MD}}(\Omega) & =\sum_{(\alpha \beta) \in \Omega} c_{(\alpha \beta)} \phi\left(\vec{r}_{\alpha \beta}\right)
\end{aligned}
$$

where $K_{i j}$ is the stiffness matrix of linear elasticity [15] and $\phi\left(\vec{r}_{\alpha \beta}\right)$ is the MD two-body potential. The coefficients $c_{(\alpha \beta)}$ equal 1 for all bonds $(\alpha \beta)$ that lie entirely within the interior of the region $\Omega$ and are non-zero only for those bonds $(\alpha \beta)$ for which both atoms $\alpha$ and $\beta$ are in the interfacial region, $\Omega$. For bonds on the surface of $\Omega, c_{(\alpha \beta)}$ is equal to the fraction of the cylindrical angle about the line $(\alpha \beta)$ that lies in $\Omega$. For example, a bond lying on the face of cell on the exterior of $\Omega$ would be weighted $c_{(\alpha \beta)}=1 / 2$, and a bond on the exposed edge of a cubic cell would be weighted $c_{(\alpha \beta)}=1 / 4$. Thus, $c_{(\alpha \beta)}$ is in some sense the fraction of bond $(\alpha \beta)$ that lies in the interfacial region. These weightings did not appear in Ref. [18], since no nearest-neighbor bonds lie on the surface of the two dimensional FE/MD (100) interfacial region for silicon.

The situation is more complicated when the empirical potential includes many-body interactions and not just pair potentials. In this case the following prescription is effective. Suppose a cluster of atoms interact through a many-body term in the potential, and some of the atoms are on the MD side of the fiducial interface while other are on the FE side. This term in the energy, and the corresponding forces, is then counted with full weight in the energy subject to the following modification. The MD potential energy is rewritten as

$$
U^{M D}=U_{\text {many-body }}^{M D}+U_{\text {pair }}^{M D}
$$




$$
\begin{aligned}
& =\left(U_{\text {many-body }}^{M D}-\bar{U}_{\text {pair }}\right)+\left(\bar{U}_{\text {pair }}+U_{\text {pair }}^{M D}\right) \\
& =U_{\text {many-body }}^{\prime}+U_{\text {pair }}^{\prime}
\end{aligned}
$$

where we have added and subtracted the same term, a sum of pair potentials that represents the attraction of the many-body term in a mean field approximation. Of course this makes no difference in the interior of the MD region, and the original $U^{M D}$ is used there, but at the FE/MD interface the two-body terms are treated differently than the many-body terms, and it does make a difference. Specifically, for copper we have used the EAM potential developed by Oh and Johnson [12] and a Morse pair potential [24].

In particular, the two-body terms are treated in the same mean-force prescription described above. The repackaged two-body term, $U_{\text {two-body }}^{\prime}$, includes both repulsive and attractive parts. It gives the correct lattice constant and in close-packed crystals it ensures structural stability. Note that this decomposition has the advantage that the many-body potential is much weaker, so the fact that it is being weighted across a broader range of distances does not cause a problem. We have validated this approach through the interface scattering methods described in Ref. [18] and Ref. [22] for Stillinger-Weber and pair potentials. Note that the well-known shortcomings of pair potentials for metals are mitigated by the greatly restricted region in which they are used.

In principle, it might be preferable to weight the many-body terms differently, e.g. by the fraction of atoms on the MD side of the fiducial surface, so that the many-body interactions go smoothly from full weight to zero weight across an interface with a thickness of twice the range of the many-body potential. At this point there are many possibilities that we have yet to explore, so further optimization may be possible. In any case, it is important to modify the interactions at the level of the potential rather than at the level of the forces in order to ensure energy conservation and translation invariance. Otherwise, there is some flexibility which is used to make the interface as seamless as possible. A slightly different prescription is given in Ref. [18] for the three-body terms in the Stillinger-Weber potential. There is no need to subtract a mean-field two-body term in this case, and they weighted all three-body interactions crossing the fiducial surface with a factor of one-half.

We note that there are other approaches to defining the FE/MD coupling which are more accurate for some applications. One methodology is Coarse-Grained Molecular Dynamics (CGMD) [22] which generalizes finite element analysis in a way that guarantees that the equations of motion transition smoothly from those of finite elements in large cells to those of molecular dynamics in atomic-sized cells. It also gives a natural prescription for the random-dissipative forces due to mode suppression in the FE region [10]. This kind of term has been shown recently to be able to prevent the pathological reflection of waves induced by the coarse-graining process [26], at least for a system in which the periphery is coarsened completely, and represented by a Green function.

Once the mesh and the multiscale equations of motion have been introduced, the next step is to choose a time integrator. We have used the velocity Verlet algorithm [14] with a typical MD time step. This integrator and time step is used throughout the simulation. In principle it would be more efficient to use a longer time step in the peripheral FE regions where the mesh is large. The effective Einstein frequency is smaller there, so a longer time step is natural; however, it was found that the use of different time integrators with 
different time steps in the FE and MD regions was problematic. It led to exponential heating at the interface due to a numerical instability [16,18]. Broughton resolved this problem by implementing a uniform time integration and time step [18]. This issue would need to be revisited if the full distributed FE mass matrix were to be used. In that case, an implicit integrator would be required [15].

\section{RESULTS}

Recently, several groups have published studies of dissipation in microresonators [4-6,27]. There has been no consensus from the experimental papers on the mechanisms of dissipation. The Roukes group has studied dissipation as a function of system size, for a range of systems all significantly larger than the systems we can model. They have found that the dissipation of the resonator $(1 / \mathrm{Q}$ where $\mathrm{Q}$ is the quality factor) is roughly proportional to the surface area-to-volume ratio; i.e. for small systems the dissipation increases as the size decreases [27]. This is contrary to the original intent of the resonators which was an attempt to reduce the dissipation by going to small sizes since the device could be made from a single crystal. The Roukes group has also made interesting counter-balanced torsional resonators where one torsional bar rotates one way while the other in the opposite direction. This eliminates much of the stress at the point of attachment of the resonator to the substrate. The Kenny group has also studied dissipation in microresonators, and they too noted a linear dependence on the surface area-to-volume ratio [5]. More recently, Craighead's group at Cornell has fabricated an array of resonators on a chip going to substantially smaller sizes, and they found the same trend [6].

Our MEMS simulations have focused on the role of atomistic phenomena in sub-micron devices. Such effects would be missed by conventional finite elements, because the constitutive models are developed at large length scales where more data are available. The surface effects are negligible at these scales, so they are not included in the models. One of the results of the multiscale modeling approach has been to produce new constitutive equations that include atomistic effects. We have simulated micro-resonators with various sizes, defect concentrations and temperatures, for comparison, with the oscillating bar up to $0.2 \mu \mathrm{m}$ in length. All of the devices have the same aspect ratio, 25:2:1, and the same substrate geometry (see Fig. 1) . A typical configuration would consist of 1.6 million MD atoms, of which 0.5 million are in the resonating bar, together with 0.2 million FE nodes in the periphery. The simulation of the resonator motion is conducted as follows. The atoms are initially arranged in a single crystal lattice of silicon or stoichiometric quartz in the desired device geometry. The system is brought to thermal equilibrium in roughly 100,000 time steps by a velocity renormalization thermostating procedure. Then the resonator is offset into one of its flexural normal modes of oscillation, and released. Once released, the thermostat is decoupled, and no additional energy is put into the system. An alternative approach that would more closely mimic the experiments would be to drive the resonator at a variety of different external frequencies and measure the response curve. Such an approach would be much more costly in terms of computation time, because the motion of the system would have to be evolved until all of the transients

have died out. This can be many oscillations. The simulation of a single oscillation of a one million atom resonator takes two weeks of supercomputer time, so it would not be possible 


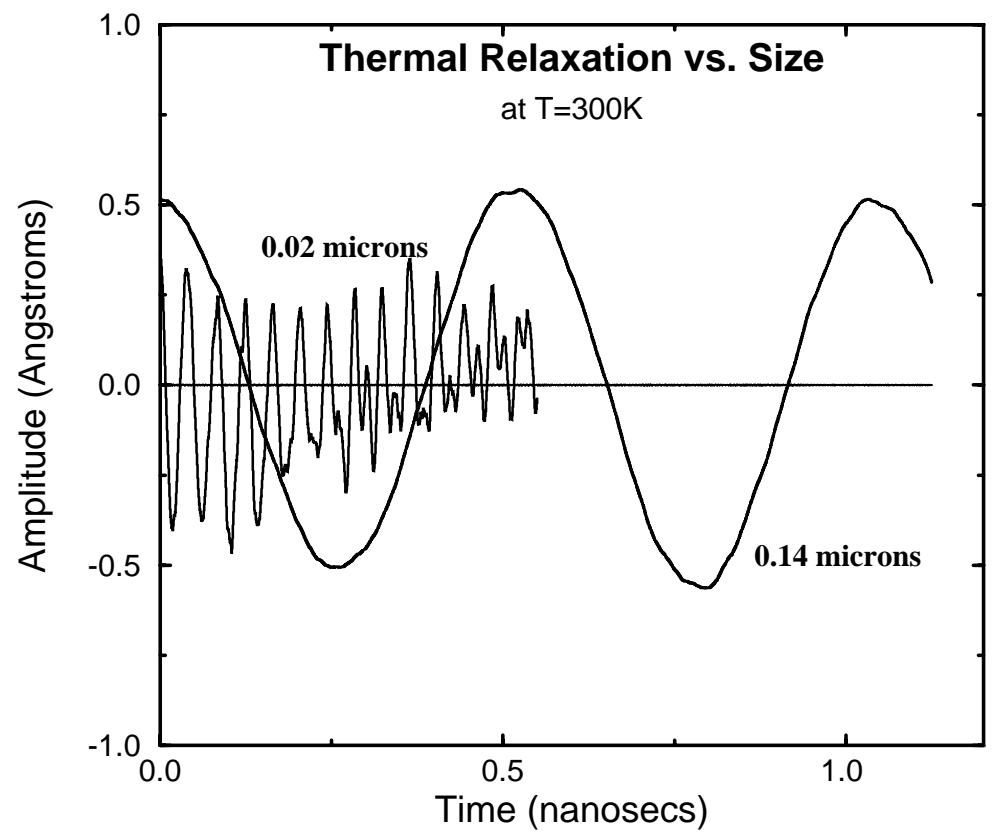

Figure 4: A comparison of the simulated oscillations of two microresonators with the same aspect ratio: the one with the short period oscillations was $0.02 \mu \mathrm{m}$ long while the other was $1.4 \mu \mathrm{m}$ long. Note the marked dissipation in the smaller system. (See Ref. [21])

to conduct a meaningful simulation of a large, driven system. Instead, we have chosen to use initial conditions based on a continuum calculation where we predict transients are negligible. This is then verified using small systems at low temperature which have a very harmonic response, and it would be possible to identify any transients that are present.

Various properties of the resonator have been studied, and many of the results have been reported elsewhere [21]. Fig. 4 shows how the oscillator rings as a function of time when plucked in flexural mode. Note that relatively large deflections of the resonator are possible, as great as $0.2 \%$, due to the increased compliance of the microscopic devices. The response of the oscillator at $300 \mathrm{~K}$ shows marked effects of anharmonicity [25]. There is a pronounced frequency doubling effect in the smaller oscillator, and even in the first few periods of the larger oscillator there are clear departures from a sinusoidal oscillation.

The mode mixing is most apparent in the Fourier transform of the oscillations of the small device shown in Fig. 4. We have used a windowed Fourier transform to compute the energy transfer from the fundamental mode into higher modes as a function of time, and fit to exponential decay for a range of systems. The result is that the $\mathrm{Q}$ of the small oscillator shown in Fig. 4 is 300 at room temperature, and the dissipation, 1/Q, is approximately proportional to the surface area-to-volume ratio. By the end of the run a significant amount of the first harmonic (as well as a bit of the second) has mixed into the spectrum. This is not the case for an identical simulation run at $\mathrm{T}=10 \mathrm{~K}$, where only the fundamental mode is present (not shown) [25]. Note that the room temperature behavior is in stark contrast to the classic Fermi-Pasta-Ulam behavior characteristic of low temperature 
systems that are only slightly perturbed from an integrable model [28]. Our simulations of the resonators show appreciable relaxation at room temperature.

This behavior would not be predicted from continuum elastic theory based on macroscopic constitutive laws. The anharmonic response has been shown to be the result of surface effects, which are negligible at large length scales. The degradation of the Q-value of small resonators is also due to a surface effect, not the bulk thermoelastic and phonon-phonon processes that are important in large single crystal resonators [29]. The reduction of $\mathrm{Q}$ for small resonators was observed in the early experiments on microresonators $[4,5]$, where it has been attributed to flawed surfaces. We have found that show that even for initially perfect single crystal devices, atomistic effects cause a significant degradation in Q at room temperature [21].

\section{CONCLUSION}

The multiscale simulation techniques we have developed have proven effective in the study of a variety of systems at the sub-micron scale. In this Article we have shown how the technique may be extended to the atomistic simulation of metals, which in general require many-body empirical potentials. Solutions to the problems posed by the many-body character of the potential and its relatively long-range nature (compare to silicon where the techniques were originally developed) have been given.

We have also discussed how multiscale simulations have been used to search for new effects arising in embedded nanomechanics. We have discussed application to sub-micron MEMS systems, where departures from the macroscopic behavior are due to atomistic surface effects. The important role of surface effects at the nanoscale has been noted in many systems, and concurrent multiscale modeling offers a powerful tool to study this seemingly anomalous behavior.

\section{ACKNOWLEDGMENTS}

We owe many thanks to Jeremy Q. Broughton with whom this work was begun. Support has been provided by DARPA. Supercomputing resources at the Maui High Performance Computing Center and the Wright-Patterson Major Shared Resource Center have been provided through a DoD HPCMO Grand Challenge award. This work was

performed in part under the auspices of the U.S. Department of Energy by the University of California, Lawrence Livermore National Laboratory, under Contract No. W-7405-Eng-48.

\section{REFERENCES}

1. See for example K. E. Drexler, Nanosystems: molecular machinery, manufacturing and computation, (Wiley, New York, 1992).

2. I. Goldfarb, P. T. Hayden, J. H. G. Owen and G. A. D. Briggs, Phys. Rev. B 56, 10459 (1997).

3. C. T.-C. Nguyen, L. P. B. Katehi, and G. M. Rebeiz, "Micromachined devices for wireless communications," Proc. IEEE 86, 1756 (1988). 
4. A. N. Cleland and M. L. Roukes, Appl. Phys. Lett. 69, 2653 (1996); See also http://www.cmp.caltech.edu/ roukes/images/mrfm.jpg.

5. T. D. Stowe, K. Yasumura, T. W. Kenny, D. Botkin, K. Wago, and D. Rugar, Appl. Phys. Lett. 71, 288 (1997).

6. D. W. Carr, S. Evoy, L. Sekaric, H. G. Craighead, and J. M. Parpia, Appl. Phys. Lett. 75, pp. 920-2 (1999).

7. G. Medieros-Ribeiro, A. M. Bratkovski, T. I. Kamins, D. A. A. Ohlberg and R. S. Williams, Science, 279, 353 (1998).

8. J. Belak, J. Comp.-Aided Mater. Design 5, 193 (1998).

9. M. J. Allen, E. M. Bradbury, R. Balhorn, Scanning Microscopy, 10, 989 (1996).

10. R. E. Rudd and J. Q. Broughton, Phys. Stat. Sol. (b) 217, 251-91 (2000).

11. M. S. Daw and M. I. Baskes, Phys. Rev. B 29, 6443 (1985).

12. D. J. Oh and R. A. Johnson, "Embedded Atom Method for Close-packed Metals," in Atomistic Simulation of Materials: Beyond Pair Potentials, V. Vitek and D. J.

Srolovitz, eds., Proc. of Int. Symp. on Atomistic Sim. of Mater., Sept. 25-30, 1988 (Plenum, Oxford, 1989), p. 233.

13. M. W. Finnis and J. E. Sinclair, Philos. Mag. A 50, 45 (1984).

14. M. P. Allen and D. J. Tildesley, Computer Simulation of Liquids, (Clarendon Press, Oxford, 1987).

15. See for example: O. C. Zienkiewicz and R. L. Taylor, The Finite Element Method, 4th ed. (McGraw-Hill, New York, 1991), Vol. II.

16. S. Kohlhoff, P. Gumbsch, and H. F. Fischmeister, Philos. Mag. A 64, 851 (1991).

17. E. B. Tadmor, M. Ortiz, and R. Phillips, Philos. Mag. A 73, 1529 (1996).

18. J. Q. Broughton, N. Bernstein, E. Kaxiras, and F. F. Abraham, Phys. Rev. B 60, 2391 (1999).

19. F. H. Stillinger and T. A. Weber, Phys. Rev. B 31, 5262 (1985).

20. A. Nakano, B. Lingsong, P. Vashishta, and R. K. Kalia, Phys. Rev. B 49, 9441 (1994).

21. R. E. Rudd and J. Q. Broughton, J. Model. and Sim. of Microsys. 1, 26 (1999).

22. R. E. Rudd and J. Q. Broughton, Phys. Rev. B 58, R5893 (1998).

23. R. E. Rudd, Proc. of 2000 Intl. Conf. on Modeling and Simulation of Microsystems (MSM2000), March 2000, San Diego, M. Laudon and B. Romanowicz, eds. (Computational Publications, Boston, 2000), p. 465.

24. K. Maekawa and A. Itoh, Wear 188115 (1995).

25. J. Q. Broughton, C. A. Meli, P. Vashishta, and R. K. Kalia, Phys. Rev. B 56, 611 (1997).

26. W. Cai, M. de Koning, V. V. Bulatov and S. Yip, Phys. Rev. Lett. 85, 3213 (2000).

27. P. Mohanty, D. A. Harrington, K. L. Ekinci, Y. T. Yang, M. J. Murphy and M. L. Roukes, "Intrinsic Dissipation in High Frequency Micromechanical Resonators," submitted to Phys. Rev. B, 2000.

28. E. Fermi, J. Pasta, and S. Ulam, "Studies of Nonlinear Problems," Los Alamos Report LA-1940 (1955).

29. V. B. Branginsky, V. P. Mitrofanov and V. I. Panov, Systems with Small Dissipation, (Univ. Chicago Press, Chicago, 1985). 